\author{
Biagio Moretti \\ Raffaele Garofalo \\ Vittorio Patella \\ Gian Lorenzo Sisti \\ Margherita Corrado \\ Elyazid Mouhsine
}

\section{Extracorporeal shock wave therapy in runners with a symptomatic heel spur}

Received: 19 February 2005

Accepted: 15 June 2005

Published online: 14 December 2005

(C) Springer-Verlag 2005
B. Moretti · V. Patella - G. L. Sisti

M. Corrado

Department of Clinical Methodology and Surgical Technique, Orthopedics Section II, University of Bari, Bari, Italy

R. Garofalo · E. Mouhsine $(\square)$ Department of Orthopaedic and Traumatology, University Hospital, OTR-BH 14, CHUV,

1011 Lausanne, Switzerland

E-mail: Elyazid.Mouhsine@hospvd.ch

Tel: + 41-21-3142791

Fax: +41-21-3142800

\begin{abstract}
The aim of this paper is to assess the benefit to treat plantar fasciitis with low-dose energy extracorporeal shock wave therapy (ESWT) and the efficacy of such treatment to abate the painful symptoms allowing a rapid return to the running activity. Our study included 54 running athletes treated for plantar fasciitis associated with a heel spur who received four sessions (once weekly) of low-dose ESWT, and followed prospectively on average 45 days, 6 and 24 months after their last session. The clinical results were excellent in $59 \%$ of cases, good in $12 \%$, satisfactory in $21 \%$ and distinctly unsatisfactory in $8 \%$. No patient was observed a significant modification of the heel spur at the follow-up X-ray. The ultrasound
\end{abstract}

examination at 24 months showed a disappearance of the inflammation signs in $61 \%$ of cases. A strong correlation between ultrasound improvement and clinical results were found. Low-energy ESWT seems to be a good mean to treat plantar fasciitis in runners with a $71 \%$ of good or excellent results and a persistent improvement lasting 24 months. A randomized multicentric study seems to be necessary to define the type of energy that should be used in the future to treat plantar fasciitis, in particular in the athletic patients, to allow a faster return to sports activities.

Keywords Extracorporeal shock wave therapy ' Heel spur ·

Plantar fasciitis · Shockwave

\section{Introduction}

The chronic proximal plantar fasciitis is associated with a painful, often intolerable and invariably incapacitating symptoms in the inferior surface of the heel, that characteristically worse on first arising in the morning or after a period of rest and decreases as they start to walk.

Pathology studies done on surgically removed specimens demonstrated microtears of fascia, collagen necrosis, angiofibroblastic hyperplasia and chondroid metaplasia [8]. On average, $50 \%$ of the patients with heel pain have an inferior calcaneal bone spur adjacent to the plantar fascial enthesis $[2,7,15,17]$. This spur may be a reaction to altered fascial mechanics, inflammation or mechanical stimulation of the plantar soft tissue [3, 15]. The runners at recreational jogging or at competitive level may be susceptible to suffer this problem [4]. A wide range of treatments have been suggested, consisting of decreasing activity, heel cord stretching, night splints, anti-inflammatory drugs, heel cups, pads and orthotics [4], which in most cases can resolve the problem, although the symptoms can persist for 10-12 months.

More recently extracorporeal shock wave therapy (ESWT) has been used in the treatment of a number of musculoskeletal condition, including plantar fasciitis. The rational of this treatment is the stimulation of soft tissue healing, reduction of calcification, inhibition of pain receptors or denervation to achieve pain relief $[6,10,18]$. The efficacy of this treatment seems to be dependent on doses and regimes [19].

The aim of this paper is to assess the benefit to treat plantar fasciitis with low-dose energy ESWT and the efficacy of such treatment to abate the painful symptoms allowing a rapid return to the running activity. 


\section{Materials and methods}

Our trial included 54 running athletes treated for plantar fasciitis between January 2000 and January 2002. There were 20 competitive level runners and 34 recreational joggers, with a mean age of 35.2 years (range 30-42). The symptoms were unilateral in all the cases. Our inclusion criteria were the following: the presence of a chronic pain (at least 6 months of duration) at the proximal insertion of the plantar fascia in the inferior heel, which failed to respond to a trial of conservative treatment including medical, physical therapy, local injection and orthotic devices. The presence of heel spur on the lateral X-ray view of foot before start of the treatment was our second criteria of inclusion. An ultrasound evaluation using an ESAOTE AU 5 Genova device with linear $50 \mathrm{MHz}$ probe was performed in the region of maximal tenderness by a physician participant in the study. The treatment protocol consisted in a course of four sessions (once weekly), with 2,000 pulses being delivered at each session at an average of $0.04 \mathrm{~mJ}$ $\mathrm{mm}^{2}$, using a electromagnetic lithotripter (MINILITH SL1 by STORZ MEDICAL) with cylindrical coil, parabolic focus and ultrasound in-line scanning.

No local anesthetic was used during session of treatment. We aimed the device directly at the medial tubercle of the calcaneus, at the proximal insertion of the plantar fascia or the calcaneal spur, around the predetermined point of medial tenderness.

The patients were invited to come back at an average of 45 days, 6 and 24 months since their last ESWT session. At follow-up we have evaluated the pain levels using the VAS scale (where ten represented unbearable pain and zero absence of pain). The values

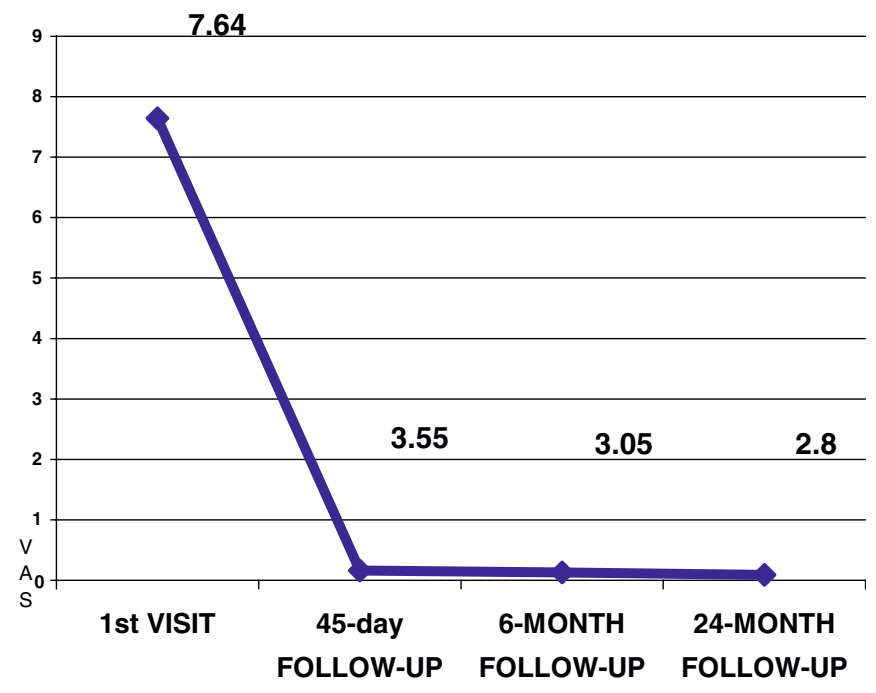

Fig. 1 The graph shows the mean value of pain $(V A S)$ at the time 0 (pretreatment time) and at the follow-up time taken were the average score at rest, during the first morning steps and after running or jogging. At each follow-up visit, the dolorimeter was applied to the baseline (pretreatment) and the patient was asked to rate the VAS. At the same intervals of time a lateral weight-bearing X-ray of the foot and ultrasound evaluation using an ESAOTE AU 5 Genova device with linear $50 \mathrm{MHz}$ probe was performed.

We defined the clinical results as follows: excellent, patients who reported a subjective decrease in pain ranging from 100 to $70 \%$; very good, a decrease from 70 to $30 \%$ (VAS); satisfactory a decrease of less than $30 \%$ (VAS); and unsatisfactory results in which the clinical picture was unchanged or worse.

We checked our assumption using Student's $t$ test for dependent samples, and we used Pearson's correlation coefficient to measure the linear association between two characters.

\section{Results}

Before start of the treatment, the ultrasound evaluation showed signs of inflammation such as, oedema or bursitis around the proximal insertion of the plantar fascia, in all cases.

The mean VAS before treatment was 7.64 (SD 1.48). At the 6-week follow-up, the mean VAS was 3.55 (SD 3.06 ), at 6 months the value was 3.05 (SD 2.56) and at 24 months 2.8 (SD 3.30), Fig. 1.

Based on the aforementioned parameters, we had excellent results in $59 \%$ of cases (32 patients), very good results in $12 \%$ (seven patients), satisfactory results in $21 \%$ (11 patients) and distinctly unsatisfactory results in $8 \%$ (four patients), Fig. 2.

Fifty patients were able, although a different level of satisfactory, to resume them athletic activities as high as before this treatment at a mean time of 34 days (range 28-45 days) after starting the treatment.

The remaining four patients were not able to resume athletic activities because of persistence of symptoms. Three of these patients underwent surgical management consisting in a plantar fascia release and removal of the inflamed necrotic fascia with a satisfactory result; the last patient refused any surgical treatment; it was a recreational jogger at a low level and continued occasionally his jogging using heel cup and taking sometimes, anti-inflammatory no-steroidal drugs.

In no case, fragmentation or disappearance of the heel spur was observed at the X-ray performed during the follow-up time.

The ultrasound evaluation at 24 months showed that 21 patients $(39 \%)$ still showed evidence of inflammation, but 33 patients $(61 \%)$ showed a complete disappearance of the inflammatory signs. In the group in which we had excellent and very good results (39 patients 71\%), 


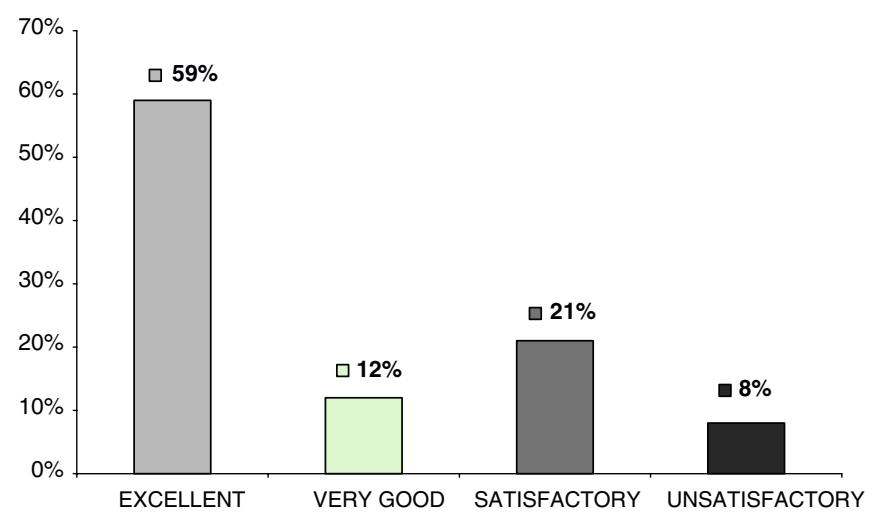

Fig. 2 The results obtained with the treatment of low-energy ESWT

(28 patients $71 \%$ ) showed ultrasound evidence of improvement with no sign of inflammation, while in 11 patients $(28 \%)$ clear evidence of inflammation still persisted. In the group in which we had discrete and poor results (15 patients, $29 \%$ ), only in six patients $(40 \%)$ we do not found evidence of inflammation, while in nine patients $(60 \%)$ the inflammation persisted. We observed a strong correlation between improvements evidenced by ultrasound at the 24-month follow-up and improvements in reported pain on the VAS scale $(r=0.30$ statistically significant for $P=0.001$ ), Fig. 3 .

\section{Discussion}

The plantar fascia plays a dominant role, during weightbearing activities, contributing a larger proportion of maintenance of the arch than the spring ligament or plantar ligaments [12]. The calcaneum attachment site is subjected to tensile stress with weight bearing and locomotion. The runners, at whatever level can be predispose to develop plantar fasciitis, due to overuse, training errors or footwear. Due to the repetitive nature of walking activities, plantar fasciitis can become a chronic problem, characterized by a chronic inflammation and microtears in the plantar fascia, as evidenced in the three cases who were operated on, in our series.

Inflammation of the plantar fascia, and the resulting bursitis, seems to be responsible of heel pain, while the heel spur do not have nerve endings of its own and is thus asymptomatic. The heel spur is merely an associated finding that indicates the presence of long-standing bursitis and plantar fasciitis, but in some cases, it can be large enough to be causing mechanical pain or compressing the first branch of the lateral plantar nerve [4].

In our series using ultrasound, the presence of inflammatory signs was noted at the pretreatment time in all the cases, and the reduction of these signs at follow-up was well correlated with good results.
The presence of heel spur was a criteria of inclusion in our study, so it was impossible to define the correlation between this finding and plantar fasciitis. In the literature, an incidence between $50 \%[2,15]$ and $65 \%[13]$ is reported. On the other hand, not all patients with heel spurs have a confirmed diagnosis of chronic proximal plantar fasciitis [2]. The reason to insert the presence of heel spur into the inclusion criteria was to have a group as homogeneous as possible, and to verify at the end of treatment the fate of heel spur and an eventually correlation with the results observed. Our results are similar to that reported by Lee et al. [13], which observed no modification of heel spur at the last of treatment, confirming that the presence of the heel spur do not have any correlation with the final outcome.

On the other hand, we have found a strong correlation between the disappearance of inflammatory signs at the ultrasound examination and the final outcome. This finding seems to confirm the anti-inflammatory effect of ESWT [6], but do not help to understand the causes of $8 \%$ of unsatisfactory results found in our series. Some authors, who using low-energy ESWT [10, 11, 14, 20, 21], has found that the pressure of bone marrow oedema within the calcaneus, demonstrated by bone scan and overall by MRI was highly predictive for a satisfactory final outcome. Nevertheless, the area of the fascial attachment at the medial calcaneal tubercle may show
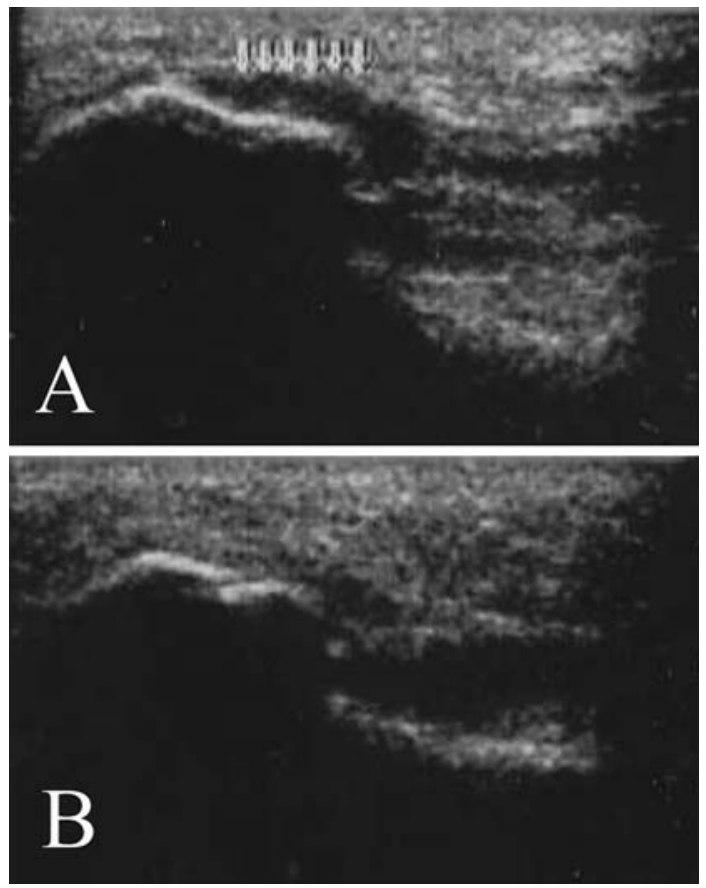

Fig. 3 a An ultrasound examination performed before starting ESWT treatment showing evidence of acute bursitis around the proximal insertion of the plantar fascia. $\mathbf{b}$ The same investigation performed in the same subject, which reported an excellent result at 24-month follow-up, shows the absence of signs of inflammation 
an increased uptake at bone scan indicative of a focal osseous stress reaction $[9,21]$. We do not have any experience with these types of examens to study this injury, but at the light of literature.

We can speculate that overall in competitive athletes suffering of plantar fasciitis may be necessary to use MRI, so a rationale treatment can be planned.

In general, our study, although performed on a particular category of subjects, shows results similar to the ones of previous studies using low ESWT in treatment of plantar fasciitis $[1,5,14,16,18]$. Speed et al. [19] in a double-blind randomized controlled trial has found no treatment effect of low-dose ESWT to treat plantar fasciitis, but the treatment was applied monthly rather than weekly interval as in recommended protocol.

Recently, the use of high energy ESWT has received FDA approval specifically for the treatment of plantar fasciitis, following an evaluation period involving seven research institutes and including double-blind placebocontrolled trials, with a $76 \%$ success rate after just one treatment [16]. Lee et al. [13] reported an $80 \%$ of success using one and sometimes two sessions of treatment. The advantage of high energy ESWT is surely the short time of treatment, in fact, as aforementioned, sometimes a single session can be sufficient and curative allowing a faster resumption of sports activities. The problem with this type of treatment is the pain during the session of treatment requiring a conscious sedation or ankle block anesthesia [13].

In conclusion, low-energy ESWT seems to be a good mean to treat plantar fasciitis in runners, with a $71 \%$ of good or excellent results and a persistent improvement lasting 24 months. The ultrasound improvements of inflammatory signs match clinical findings in $61 \%$ of cases at subsequent follow-up. The routine use of MRI at the time of diagnosis can be suggested to probably better define the patients who will beneficiate of ESWT treatment, allowing overall to the athletes a less time consuming. A randomized multicentric study seems to be necessary to define the type of energy that should be used in the future to treat plantar fasciitis, in particular in the athletic patients, to allow a faster return to sports activities. It will be interesting too to compare this technique with the others as surgery, steroid infiltration or radiotherapy. Steroid infiltration is still unsatisfactory. Radiotherapy is limited by the risk of the radioinductive sarcoma and surgery can be used by echec of ESWT treatment.

\section{References}

1. Abt T, Hopfermuller W, Mellerowicz H (2002) Shock wave therapy for recalcitrant plantar fasciitis with heel spur: a prospective a randomized placebo-controlled double-blind study. Orthop Ihre Grenzgeb 140(5):548-554

2. Amis J, Jennings L, Graham CE (1998) Painful heel syndrome: radiographic and treatment assessment. Foot Ankle 9:91-99

3. Barret SL, Day SV, Pignetti T, Egly BR (1995) Endoscopic heel anatomy: analysis of 200 fresh frozen specimens. J Foot Ankle Surg 34:51-56

4. Baxter DE, Yingas C (1995) The foot in running. J Am Acad Orthop Surg 3:136-145

5. Buch M, Knor U, Fleming L, et al (2002) Extracorporeal shockwave therapy in symptomatic heel spurs - an overview. Orthopade 31(7):637-644

6. Delius M (1994) Medical applications and bioeffects of extracorporeal shock waves. Shock Waves 4:55-72

7. Furey JP (1975) Plantar fasciitis: the painful heel syndrome. J Bone Joint Surg 57A:672-674
8. Gill LH (1997) Plantar fasciitis diagnosis and conservative management. J Am Acad Orthop Surg 5:109-115

9. Graham CE (1983) Painful heel syndrome: rationale of diagnosis and treatment. Foot Ankle 3:261-267

10. Grasel RP, Schweitzer ME, Kovalovich AM, et al (1999) MR imaging of plantar fasciitis: edema, tears, and occult marrow abnormalities correlated with outcome. Am J Roentgenol 173:699-701

11. Ham PS, Strayer S (2002) Shock wave therapy ineffective for plantar fasciitis. J Fam Pract 51(12):1017-1022

12. Huang CK, Kitaoka HB, An KN et al (1993) Biomechanical evaluation of longitudinal arch stability. Foot Ankle 14:353-357

13. Lee GP, Ogden JA, G Lee Cross (2003) Effect of extracorporeal shock waves on calcaneal bone spurs. Foot Ankle Int 23:301-308

14. Maier M, Steinborn M, Schmitz C, et al (2000) Extracorporeal shock wave application for chronic plantar fasciitis associated with heel spurs: prediction of outcome by magnetic resonance imaging. J Rheumatol 27:2455-2462

15. McCarthy JJ, Goreecky GE (1979) Anatomical basis of inferior calcaneal lesions: a cryomicrotomy study. J Am Pediatr Assoc 69:527-536

16. Ogden JA, Alvarez R, Levitt R, Cross GL, Marlow M (2001) Shockwave therapy for chronic proximal plantar fasciitis. Clin Orthop 387:47-59

17. Onwuanyo ON (2000) Calcaneal spurs and plantar heel pad pain. Foot 10:182185

18. Rompe JD, Hopf C, Nafe B, Burger R (1996) Low-energy extracorporeal shock wave therapy for painful heel: a prospective controlled single-bind study. J Orthop Trauma Surg 115:75-79

19. Speed CA, Nichols D, Wies J, et al (2003) Extracoropreal shock wave therapy for plantar fasciitis. A doubleblind randomised controlled trial. J Orthop Res 21:937-940

20. Steinborn M, Heuck A, Maier M, Schnarkowski P, Scheidler J, Reiser M (1999) MRI of plantar fasciitis. Rogo Fortschr Geb Roentgenstr 170:41-46

21. Tudor GR, Finlay D, Allen MJ, Belton J (1997) The role of bone scintigraphy and plain radiography in intractable plantar fasciitis. Nucl Med Commen 18:853-856 